\title{
Autism spectrum disorder and Li-Fraumeni syndrome: purely coincidental or mechanistically associated?
}

\author{
Michaela Kuhlen ${ }^{1 *}$ D, Julia Taeubner ${ }^{1}$, Dagmar Wieczorek ${ }^{2}$ and Arndt Borkhardt ${ }^{1}$
}

\begin{abstract}
Background: Autism spectrum disorders (ASDs) are neurodevelopmental disorders with impaired social interactions and communication and restrictive, repetitive patterns of behaviors, interests, and activities. A recent epidemiological study suggests that children with ASD might have an increased cancer risk.

Case presentation: The 14.5-year-old boy, previously diagnosed with ASD, was referred with persistent bone pain. Diagnostic work-up confirmed diagnosis of acute lymphoblastic leukemia (ALL); cytogenetic analysis revealed low hypodiploid karyotype with a mutation (c.733G>A, p.Gly245Ser, rs28934575) in TP53 in the leukemic blasts. By Sanger sequencing, the presence of this mutation in the germline was subsequently confirmed and, thus, diagnosis of Li-Fraumeni syndrome (LFS) was made. His family history was remarkable with two siblings with intellectual disability and a mother who has died of premenopausal breast cancer.

Conclusions: Some of the oncogenes and tumor suppressor genes causing cancer susceptibility syndromes overlap with those involved in autism. This functional overlap between autism and cancer is novel and particularly compelling. The surprising coincidence of LFS and ASD in our patient raises the question whether this is purely coincidental or mechanistically associated.
\end{abstract}

Keywords: Autism spectrum disorder, Li-Fraumeni syndrome, Hypodiploid leukemia

\section{Findings}

Autism spectrum disorder (ASD) is a group of neurodevelopmental disorders with impaired social interactions and communication and restrictive, repetitive patterns of behaviors, interests, and activities. The prevalence of ASDs in the general population has been estimated to be $1 \%$ worldwide, with a high male to female ratio. This number seems even higher in children with around 1 in every 68 children (Report 2014 of The Centers for Disease Control and Prevention's Autism and Developmental Disabilities Monitoring Network). A recent epidemiological study suggests that children with ASD might have an increased cancer risk [1].

We report a boy of non-consanguineous parents, previously diagnosed with ASD at the age of 13 years. His

\footnotetext{
* Correspondence: michaela.kuhlen@med.uni-duesseldorf.de

'Department of Pediatric Oncology, Hematology and Clinical Immunology, University Children's Hospital, Medical Faculty, Heinrich Heine University, Moorenstr. 5, 40225 Duesseldorf, Germany

Full list of author information is available at the end of the article
}

family history was remarkable with an older sister with intellectual disability not further specified, a younger brother with profound and multiple disabilities, and a mother who has died of premenopausal breast cancer. The boy was referred to our department at the age of 14.5 years with persistent bone pain. MRI revealed leukemic infiltration of the femura and spine, and further diagnostic work-up confirmed the diagnosis of ALL. Cytogenetic analysis revealed a low hypodiploid karyotype with a deletion of $17 \mathrm{p}$ and molecular analysis an additional mutation (c.733G >A, p.Gly245Ser, rs28934575) in TP53 (NM_000546.5) in the leukemic blasts. The mutation was subsequently confirmed by conventional Sanger sequencing in fibroblast-derived germline DNA; thus, the diagnosis of Li-Fraumeni syndrome (LFS, OMIM \#151623) was made. The boy was treated according to the high-risk protocol of the AIEOP-BFM 2009 trial and is, 24 months after diagnosis, doing well under maintenance therapy. 
This report documents the surprising coincidence of LFS and ASD, thus, raising the question whether this is purely coincidental or mechanistically associated.

A high number of ASDs are hereditary cases [2], and there is mounting evidence that childhood cancer has likewise a higher fraction of cases with genetic traits than previously expected [3].

Advances in high-throughput sequencing have led to greater understanding of genetic mechanisms in ASD. Several of the susceptibility genes identified in ASD patients that are also associated with cancer are involved in chromatin remodeling, genome maintenance, signal transduction pathways, histone modification, and share functions as transcription factors $[4,5]$. Some of the oncogenes and tumor suppressor genes like PTEN and NF1 are implicated in causing hereditary cancer susceptibility syndromes overlap with those involved in autism [4, 6]. Furthermore, copy number variations $(\mathrm{CNV})$ recently described in children with autism are associated with cancer predisposition genes. This functional overlap between autism and cancer is novel and particularly compelling.

Li-Fraumeni syndrome, resulting from germline mutations in the TP53 gene, represents a well-known cancer susceptibility syndrome. TP53 is a tumor suppressor gene encoding the transcription factor p53 that responds to several forms of cellular stress and, thus, plays a pivotal role in cell growth control, DNA repair, cell cycle suppression and senescence and eventually in the initiation of apoptosis. Experimental assays on the functional impact of mutant proteins have revealed different mechanisms including (1) transactivation of reporter genes in the (downstream) pathways of p53; (2) activation of genes that are independent, unrelated to, and/or repressed by the wild-type protein (gain of function; GOF); (3) induction of cell cycle arrest or apoptosis; and (4) dominant-negative effect over the wild-type protein.

Interestingly, recent studies imply, on the one hand, a negative feedback loop between TP53 and phosphatase and tensin homolog (PTEN) [7-9] and, on the other hand, a link between autism and incomplete loss of PTEN function [10]. Additionally, it was also shown that p53 gene copy ratios were increased in children with autism [11]. It might be hypothesized that ASD is mechanistically associated with LFS and, thus, might be part of the LFS phenotype.

We observed a surprising coincidence of LFS with ASD, which warrants further testing in large cooperative studies.

\section{Abbreviations}

ALL: Acute lymphoblastic leukemia; ASD: Autism spectrum disorder CNV(s): Copy number variant(s); LFS: Li-Fraumeni syndrome;

PTEN: Phosphatase and tensin homolog

\section{Authors' contributions}

MK drafted the manuscript. JF and DW critically revised the manuscript for important intellectual content. MK and $A B$ designed the project. $A B$ critically revised the manuscript for important intellectual content. All authors approved the final manuscript as submitted.

Consent for publication

Written informed consent was obtained from the father.

\section{Competing interests}

The authors declare that they have no competing interests.

\section{Publisher's Note}

Springer Nature remains neutral with regard to jurisdictional claims in published maps and institutional affiliations.

\section{Author details}

${ }^{1}$ Department of Pediatric Oncology, Hematology and Clinical Immunology, University Children's Hospital, Medical Faculty, Heinrich Heine University, Moorenstr. 5, 40225 Duesseldorf, Germany. ${ }^{2}$ Institute of Human Genetics, Medical Faculty, Heinrich Heine University, Duesseldorf, Germany.

Received: 24 February 2017 Accepted: 24 September 2017

Published online: 05 October 2017

\section{References}

1. Chiang HL, Liu CJ, Hu YW, Chen SC, Hu LY, Shen CC, Yeh CM, Chen TJ, Gau SS (2015) Risk of cancer in children, adolescents, and young adults with autistic disorder. J Pediatr 166(2):418-423 e411. doi:10.1016/j.jpeds. 2014.10.029

2. Lai MC, Lombardo MV, Baron-Cohen S (2014) Autism. Lancet 383(9920): 896-910. doi:10.1016/S0140-6736(13)61539-1

3. Zhang J, Walsh MF, Wu G, Edmonson MN, Gruber TA, Easton J, Hedges D, Ma X, Zhou X, Yergeau DA, Wilkinson MR, Vadodaria B, Chen X, McGee RB, Hines-Dowell S, Nuccio R, Quinn E, Shurtleff SA, Rusch M, Patel A, Becksfort JB, Wang S, Weaver MS, Ding L, Mardis ER, Wilson RK, Gajjar A, Ellison DW, Pappo AS, Pui CH, Nichols KE, Downing JR (2015) Germline mutations in predisposition genes in pediatric cancer. N Engl J Med 373(24):2336-2346. doi:10.1056/NEJMoa1508054

4. Crawley JN, Heyer WD, LaSalle JM (2016) Autism and cancer share risk genes, pathways, and drug targets. Trends Genet 32(3):139-146. doi:10. 1016/j.tig.2016.01.001

5. Kim YS, Leventhal BL (2015) Genetic epidemiology and insights into interactive genetic and environmental effects in autism spectrum disorders. Biol Psychiatry 77(1):66-74. doi:10.1016/j.biopsych.2014.11.001

6. Darbro BW, Singh R, Zimmerman MB, Mahajan VB, Bassuk AG (2016) Autism linked to increased oncogene mutations but decreased cancer rate. PLoS One 11(3):e0149041. doi:10.1371/journal.pone.0149041

7. Harris SL, Levine AJ (2005) The p53 pathway: positive and negative feedback loops. Oncogene 24(17):2899-2908. doi:10.1038/sj.onc.1208615

8. Hat B, Kochanczyk M, Bogdal MN, Lipniacki T (2016) Feedbacks, bifurcations, and cell fate decision-making in the p53 system. PLoS Comput Biol 12(2): e1004787. doi:10.1371/journal.pcbi.1004787

9. Napoli E, Ross-Inta C, Wong S, Hung C, Fujisawa Y, Sakaguchi D Angelastro J, Omanska-Klusek A, Schoenfeld R, Giulivi C (2012) Mitochondrial dysfunction in Pten haplo-insufficient mice with social deficits and repetitive behavior: interplay between Pten and p53. PLoS One 7(8):e42504. doi:10.1371/journal.pone.0042504

10. Spinelli L, Black FM, Berg JN, Eickholt BJ, Leslie NR (2015) Functionally distinct groups of inherited PTEN mutations in autism and tumour syndromes. J Med Genet 52(2):128-134. doi:10.1136/jmedgenet-2014-102803

11. Wong S, Napoli E, Krakowiak P, Tassone F, Hertz-Picciotto I, Giulivi C (2016) Role of p53, mitochondrial DNA deletions, and paternal age in autism: a case-control study. Pediatrics 137(4). doi:10.1542/peds.2015-1888 